\title{
Effect of extubation on diaphragmatic activity in newborns measured with transcutaneous electromyography
}

R.W. van Leuteren ${ }^{1,2}$, C.G. de Waal ${ }^{1}$, F.H.C. de Jongh ${ }^{1}$, A.H.L.C. van Kaam ${ }^{1,2}$, G.J. Hutten ${ }^{1}$

${ }^{1}$ Emma Children's Hospital, Amsterdam UMC, University of Amsterdam, Neonatology, Amsterdam, The Netherlands

${ }^{2}$ Emma Children's Hospital, Amsterdam UMC, Vrije Universiteit, Neonatology, Amsterdam, The Netherlands

\section{Introduction}

Invasive mechanical ventilation in critically ill newborns aims to restore gas exchange and to reduce work of breathing, which is mainly delivered by the diaphragmatic muscle. As the lung condition improves, mechanical ventilation is weaned and ultimately the patient is extubated, often to non-invasive respiratory support. Transcutaneous electromyography of the diaphragm (dEMG) is a non-invasive technique which can record the breathing effort with surface electrodes and can be easily applied in newborns.

\section{Objective}

To investigate the changes in breathing effort after extubation in (preterm) newborns assessed with dEMG recordings.

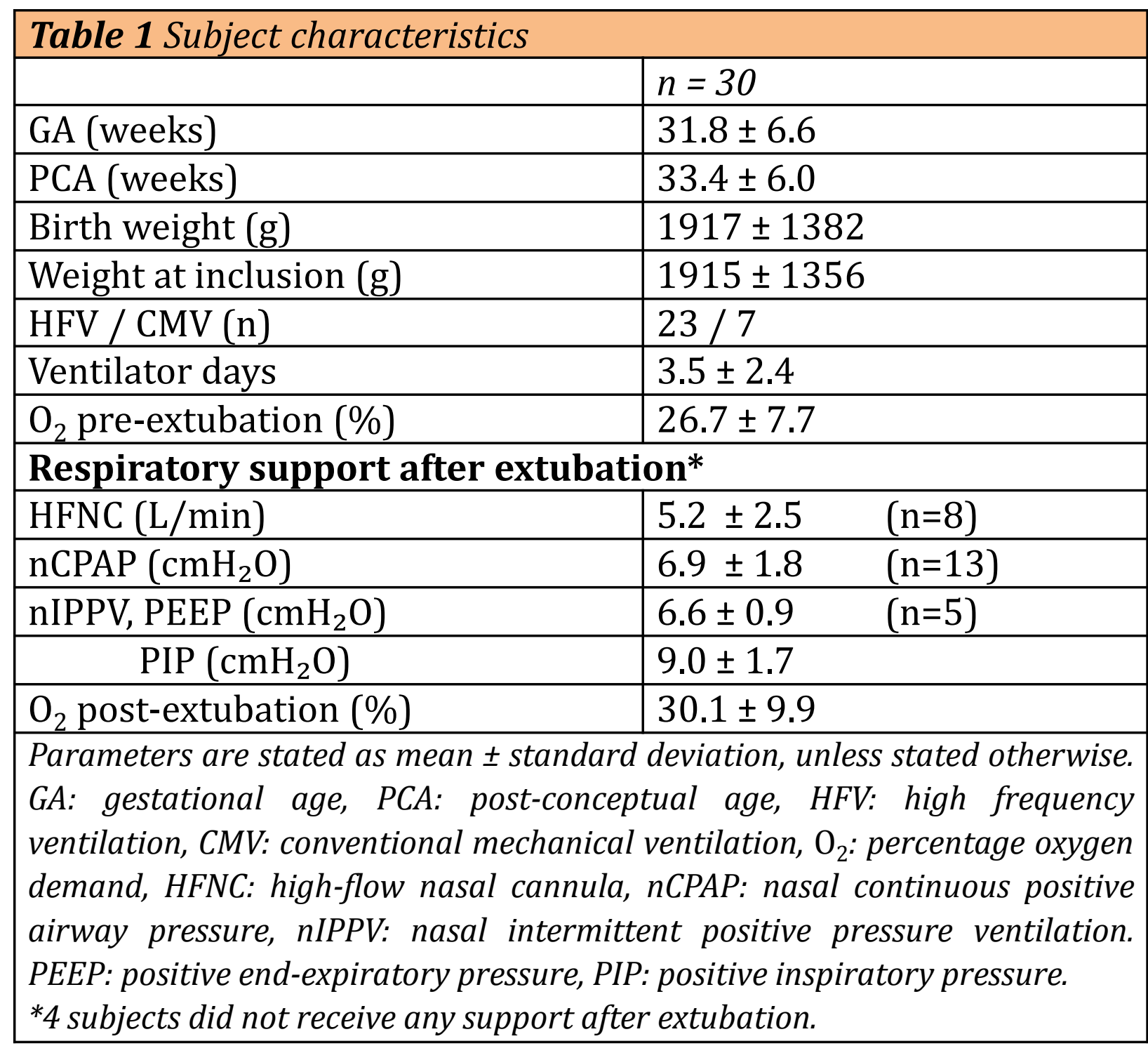

\section{Methods}

Newborns with a gestational age (GA) $\geq 26$ weeks who were ventilated $\geq 24$ hours and deemed ready for extubation were included in this prospective multi-center observational study. Extubation was performed from either conventional mechanical ventilation (CMV) or high-frequency ventilation (HFV). Spontaneous breathing was recorded with dEMG from fifteen minutes before (baseline) until three hours after extubation (Figure 1).

\section{Data analysis:}

- 60 seconds data epochs were used

- Custom-made user interface

Outcome measures

- Percentage change in dEMG amplitude, tonic and peak activity

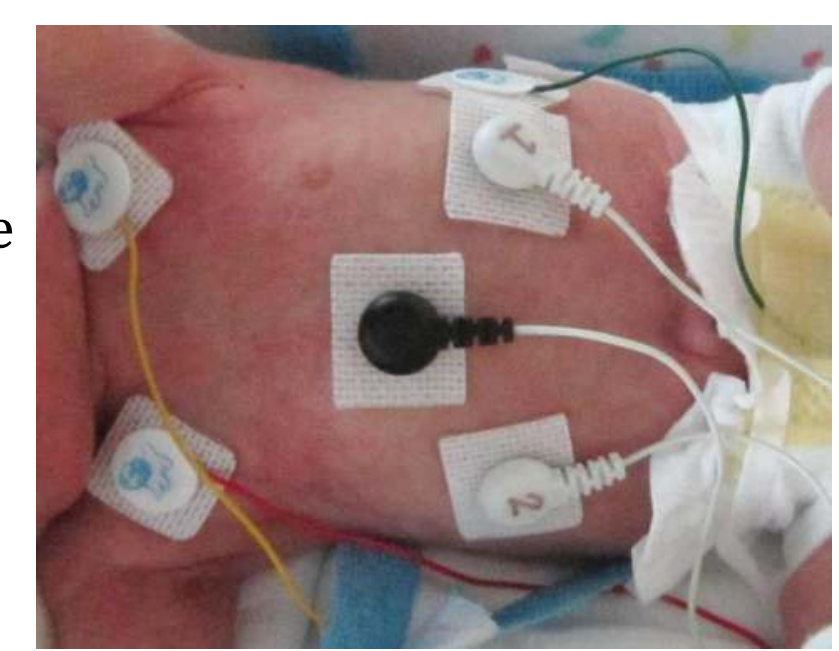

Figure 1: Measurement set-up for diaphragmatic electromyography (three electrodes: ground, 1 and 2)

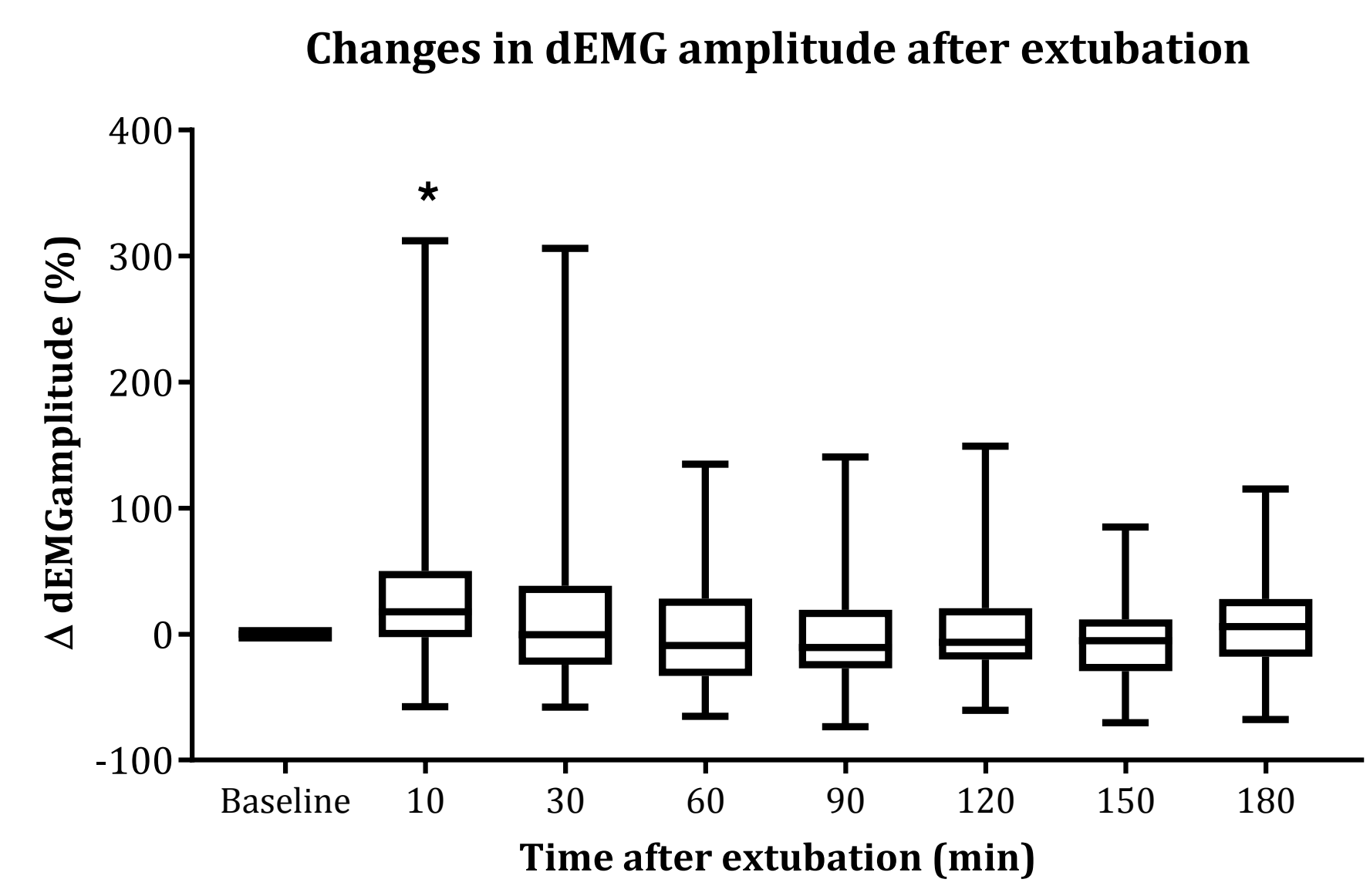

Figure 2: percentage change in amplitude of the dEMG signal at different time points after extubation compared to baseline. *significant difference compared to baseline ( $p$-value $<0.05$ ).

\section{Results}

30 newborns were included so far in this ongoing study (Table 1). One newborn failed extubation and required re-intubation within 72 hours.

The amplitude of the dEMG signal increased significantly shortly after extubation compared to baseline (Figure 2, median $+17,6 \%(+1,2+48,0 \%), p=0,02)$, primarily due to an increase in peak activity (median $+14 \%(-7,7-+43,8 \%)$ ). The tonic activity of the diaphragm did not change significantly. The dEMG amplitude returned to baseline values after thirty minutes.

\section{Conclusion}

- Diaphragmatic activity increased directly after extubation and returned to baseline values after 30 minutes in ventilated newborns.

\section{Future perspective}

In a larger cohort, the effect of extubation failure on diaphragmatic activity is being investigated. 